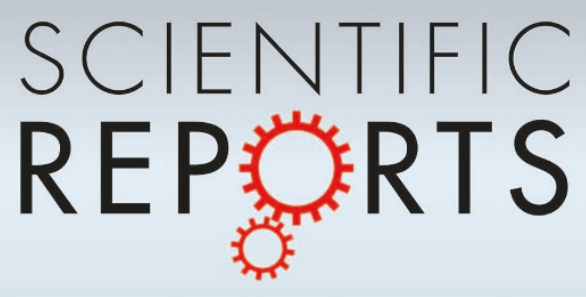

OPEN

SUBJECT AREAS:

MOLECULAR BIOLOGY

EXPERIMENTAL MODELS OF

DISEASE

Received

4 August 2014

Accepted

29 October 2014

Published

17 November 2014

Correspondence and requests for materials should be addressed to

F.J.K. (kongfeijuan@

163.com) or R.H.S.

(jqin168@hotmail.

com)

\title{
Ventricular hypertrophy blocked delayed anesthetic cardioprotection in rats by alteration of iNOS/COX-2 signaling
}

Leilei $\mathrm{Ma}^{1,2,3}$, Feijuan Kong ${ }^{2,4}$, Hongwei Ge ${ }^{5}$, Jingquan Liu' , Fangxiao Gong' ${ }^{1}$ Liang $\mathrm{Xu}^{1}$, Bangchuan $\mathrm{Hu}^{1}$ \& Renhua Sun ${ }^{1}$

'Department of Critical Care Medicine, Zhejiang Provincial People's Hospital, Hangzhou, China, ${ }^{2}$ Department of Anesthesiology, Hangzhou First People's Hospital, Nanjing Medical University, Hangzhou, China, ${ }^{3}$ Shanghai Institute of Cardiovascular Diseases, Zhongshan Hospital, Fudan University, Shanghai, China, ${ }^{4}$ Department of Endocrinology, Sir Run Run Shaw Hospital, School of Medicine, Zhejiang University, Hangzhou, China, ${ }^{5}$ Department of Urology, Peking University Shougang Hospital, Beijing, China.

The aim of the current study was to determine whether ventricular hypertrophy affects the delayed isoflurane preconditioning against myocardial ischemia-reperfusion (IR) injury. Transverse aortic constriction (TAC) was performed on male Sprague-Dawley rats to induce left ventricular (LV) hypertrophy, then sham-operated or hypertrophied rat hearts were subjected to isoflurane preconditioning $(2.1 \% \mathrm{v} / \mathrm{v}, 1 \mathrm{~h}) .24 \mathrm{~h}$ after exposure, the hearts were isolated and perfused retrogradely by the Langendorff for $30 \mathrm{~min}$ (equilibration) followed by $40 \mathrm{~min}$ of ischemia and then $120 \mathrm{~min}$ of reperfusion. The hemodynamics, infarct size, apoptosis, nitric oxide synthase (NOS), cyclooxygenase-2 (COX-2), Cleaved Caspase- 3 and production of NO were determined. We found that the hemodynamic parameters were all markedly improved during the reperfusion period and the myocardial infarct size and apoptosis was significantly reduced by delayed isoflurane preconditioning in sham-operated rats. However, such cardiac improvement induced by delayed isoflurane preconditioning was not observed in hypertrophied hearts. The expression of iNOS, COX-2 and NO was markedly enhanced, whereas Cleaved Caspase-3 activity was inhibited by delayed isoflurane preconditioning in sham-operated rats, a phenomenon was not found in TAC-control groups pretreated with isoflurane. Our results demonstrated that ventricular hypertrophy abrogated isoflurane-induced delayed cardioprotection by alteration of iNOS/COX-2 pathway.

M yocardial ischemia reperfusion (IR) injury can be reduced by multiple interventions, such as delayed ischemic/pharmacological preconditioning, in animal hearts ${ }^{1,2}$. Since the original description of delayed ischemic/pharmacological preconditioning, the mechanisms underlying this endogenous cardioprotective phenomenon have been extensively investigated ${ }^{1,2}$. Delayed ischemic/pharmacological preconditioning confers myocardial protection, at least in part, through the activation of cyclooxygenase-2 (COX-2) and COX-2-dependent synthesis of prostaglandins expression ${ }^{3-6}$. Prostanoids have been shown to exert cardioprotection against myocardial $\mathrm{IR}^{7,8}$. Besides COX-2, inducible nitric oxide synthase (iNOS) also plays an essential role in mediating the delayed cardioprotective effects of ischemic/pharmacological preconditioning ${ }^{3-6}$. The activity of newly synthesized COX-2 following preconditioning requires iNOS-derived nitric oxide (NO) whereas iNOS activity is independent of COX-2-derived prostanoids, indicating that COX-2 is located downstream of iNOS in the protective pathway of delayed preconditioning ${ }^{3,6}$.

In recent years, delayed anesthetic preconditioning has been shown to reduce myocardial apoptosis and necrosis in healthy subjects ${ }^{1,2,9}$, and the effect of delayed anesthetic preconditioning on hypertrophied rat heart remains unclear. Several studies have reported reduced protection in the late phase of pharmacological preconditioning in diseased hearts ${ }^{10,11}$. Since the late phase of pharmacological preconditioning shares similar signaling pathways, it would be reasonable that cardioprotection during the late phase would be impaired in hypertrophied heart. Left ventricular hypertrophy is accompanied by changes in the density, structure, vasodilator capacity of the coronary vasculature, metabolic and other biochemical alterations ${ }^{12,13}$. The presence of left ventricular hypertrophy on an electrocardiogram is an independent predictor of cardiovascular events, including death from coronary heart disease, sudden cardiac death, congestive cardiac failure, and stroke ${ }^{14}$. Nonetheless, no data is available with respect to delayed anesthetic preconditioning in hypertrophied myocardium. Using an established rat model of ventricular hypertrophy after permanent transverse aortic constriction (TAC), we investigated 
whether ventricular hypertrophy would affect delayed anesthetic preconditioning-induced cardioprotection. Specifically, we hypothesized that ventricular hypertrophy would abrogate delayed isoflurane preconditioning-induced myocardial protection. We also investigated possible mechanisms underlying the loss of cardioprotection by delayed isoflurane preconditioning in hypertrophied rat heart.

\section{Results}

A total of 119 rats were used for the study. 4 rats died after the operation of TAC. 39 rats were used for myocardial infarction experiments ( 1 were excluded as a result of arrhythmia duration $>2$ min and 2 were excluded as a result of LVDP $<70 \mathrm{mmHg}$ after equilibration for $30 \mathrm{~min}$ ) and 50 animals were used for immunoblotting (1 were excluded as a result of arrhythmia duration $>2$ min and 1 were excluded as a result of LVDP $<70 \mathrm{mmHg}$ after equilibration for $30 \mathrm{~min}$ ). An additional 26 rats were used for TUNEL staining ( 1 were excluded as a result of arrhythmia duration $>2 \mathrm{~min}$ and 1 were excluded as a result of LVDP $<70 \mathrm{mmHg}$ after equilibration for $30 \mathrm{~min}$ ).

TAC-induced left ventricular hypertrophy. Mean blood pressure measured in a conscious state using the tail-cuff method was higher in rats with TAC for 8 weeks than in age-matched rats with sham operation (132.4 \pm 8.9 vs. $86.4 \pm 6.1 \mathrm{mmHg}, \mathrm{P}<0.05)$, and heart rate was not significantly different $(334 \pm 30$ vs. $312 \pm 26$ beats $/ \mathrm{min}$, $\mathrm{P}>0.05)$. The ratio of heart weight: body weight $(3.9 \pm 0.3$ vs. $2.8 \pm$ $0.3 \mathrm{mg} / \mathrm{g}, \mathrm{P}<0.05)$ and the diastolic posterior wall thickness of the left ventricle assessed by echocardiography were significantly larger in rats with TAC for 8 week than age-matched rats with sham operation, indicating left ventricular hypertrophy (Table 1).

Ventricular hypertrophy abrogates the left ventricular hemodynamic improvement induced by delayed isoflurane preconditioning. The LVDP is significantly increased in rats with TAC-induced ventricular hypertrophy in baseline compared to that in sham-operated rats $(112 \pm 15$ vs. $98 \pm 11, \mathrm{P}<0.05$, Table 2$)$. The changes in LVPD and LVEDP induced by IR during reperfusion were attenuated by in vivo pretreatment with isoflurane. After $30 \mathrm{~min}$ of reperfusion, LVDP and LVEDP were all markedly improved in Sham + IR + Iso compared with that in Sham + IR group. However, this improvement did not occur in the TAC + IR + Iso compared with the TAC + IR group.

Ventricular hypertrophy abrogates the myocardial infarctsparing effect of delayed isoflurane preconditioning. As shown in Fig. 1, the infarct size was not significantly different between $\mathrm{TAC}+\mathrm{IR}$ and Sham + IR groups $(\mathrm{P}>0.05)$. The infarct size was markedly decreased in Sham + IR + Iso (29 $\pm 3 \%)$ compared with that in Sham + IR $(45 \pm 5 \%, \mathrm{P}<0.05)$, a phenomonon was not found in hypertrophied hearts (Fig. 1).

Ventricular hypertrophy inhibits the myocardial anti-apoptotic effect of delayed isoflurane preconditioning. As shown in Fig. 2, The number of TUNEL-positive nuclei expressed as a percentage of total nuclei was not significantly different between TAC + IR and Sham + IR groups $(\mathrm{P}>0.05)$, however, the reduced apoptotic nuclear conferred by delayed isoflurane preconditioning was not found in hypertrophied hearts exposed to IR.

Ventricular hypertrophy abrogates the upregulation of iNOS expression induced by delayed isoflurane preconditioning. As shown in Fig. 3A and B, total myocardial eNOS expression was not significantly different among all groups. The myocardial iNOS expression was markedly enhanced in Sham + IR + Iso compared with that in Sham + IR $(\mathrm{P}<0.05)$, which was not found in TAC + IR + Iso compared with that in TAC + IR $(\mathrm{P}>0.05)$. There was no significant difference of myocardial iNOS expression between Sham + IR and TAC + IR (Fig. 3A and B).

Ventricular hypertrophy abrogates the upregulation of COX-2 expression induced by delayed isoflurane preconditioning. As shown in Fig. 3C. The myocardial COX-2 expression was markedly enhanced in Sham + IR + Iso compared with that in Sham + IR $(\mathrm{P}<0.05)$, which was not found in TAC + IR + Iso compared with that in TAC + IR $(\mathrm{P}>0.05)$. There was no significant difference of myocardial COX-2 expression between Sham + IR and TAC + IR (Fig. 3C).

Ventricular hypertrophy abrogates the downregulation of Cleaved Caspase-3 expression induced by delayed isoflurane preconditioning. As shown in Fig. 3D. The myocardial Cleaved Caspase-3 expression was markedly decreased in Sham + IR + Iso compared with that in Sham + IR $(\mathrm{P}<0.05)$, which was not found in TAC + IR + Iso compared with that in TAC + IR $(\mathrm{P}>0.05)$. There was no significant difference of myocardial Cleaved Caspase-3 expression between Sham + IR and TAC + IR (Fig. 3D).

Ventricular hypertrophy abrogates the upregulation of NO expression induced by delayed isoflurane preconditioning. As shown in Fig. 4, the myocardial NO expression was markedly enhanced in Sham + IR + Iso compared with that in Sham + IR $(\mathrm{P}<0.05)$, which was not found in TAC $+\mathrm{IR}+$ Iso compared with that in TAC + IR $(\mathrm{P}>0.05)$. There was no significant difference of myocardial NO expression between Sham + IR and TAC + IR (Fig. 4).

\section{Discussion}

Although preconditioning with volatile anesthetics induces cardioprotection against IR injury, few studies have investigated the myocardial effect of delayed anesthetic preconditioning on animals with ventricular hypertrophy. We used TAC for 8 weeks to induce ventricular hypertrophy in male rats. We found no significant difference in left ventricular hemodynamic parameters and myocardial infarct size between sham-operated rats and rats with ventricular hypertrophy exposed to IR. Furthermore, isoflurane preconditioning produced delayed cardioprotection against reperfusion injury, in terms of decreased infarct size and improved left ventricular pump function, in sham-operated rats. Nonetheless, the delayed cardioprotection of isoflurane was lost in hypertrophied myocardium with alteration of iNOS/COX-2 signaling pathway.

Effects of TAC-induced ventricular hypertrophy on myocardial IR are poorly understood. In our current study the infarct size was not significantly increased in rats with TAC compared with sham-oprerated

Table 1 | The heart function parameters assessed by echocardiography in sham-operated and TAC-control groups 8 weeks after surgery

\begin{tabular}{lccccc} 
Group & LVEDPW $(\mathrm{mm})$ & LVEDD $(\mathrm{mm})$ & LVESD $(\mathrm{mm})$ & LVFS $(\%)$ & LVEF (\%) \\
\hline Sham & $1.5 \pm 0.1$ & $6.2 \pm 0.5$ & $2.8 \pm 0.2$ & $49.3 \pm 3.9$ & $81.2 \pm 8.2$ \\
TAC & $2.4 \pm 0.1^{*}$ & $6.8 \pm 0.6$ & $2.7 \pm 0.2$ & $38.2 \pm 3.4^{*}$ & $80.1 \pm 7.8$ \\
\hline
\end{tabular}

The heart function parameters assessed by echocardiography in sham-operated and TAC-control groups 8 weeks after surgery. LVEDPW: left ventricular end-diastolic posterior wall thickness; LVEDD: left ventricular end diastolic diameter; LVESD: left ventricular end systolic diameter; LVFS: left ventricular fractional shortening; LVEF: left ventricular ejection fraction. $* P<0.05$ vs. Sham group. $n=57$ hearts in sham group; $n=58$ hearts in TAC group. 
Table 2 | The effect of delayed isoflurane preconditioning on left ventricular hemodynamic parameters in rat hearts exposed to ischemiareperfusion

\begin{tabular}{|c|c|c|c|c|c|}
\hline \multirow[b]{2}{*}{ Group } & \multirow[b]{2}{*}{ Baseline } & \multicolumn{4}{|c|}{ Reperfusion } \\
\hline & & $30 \mathrm{~min}$ & $60 \mathrm{~min}$ & $90 \min$ & $120 \mathrm{~min}$ \\
\hline \multicolumn{6}{|c|}{ LVDP, $\%$ of baseline $(\mathrm{mmHg})$} \\
\hline Sham & $100(98 \pm 11)$ & $94 \pm 8$ & $92 \pm 8$ & $85 \pm 7$ & $83 \pm 7$ \\
\hline Sham $+\mathbb{R}$ & $100(101 \pm 12)$ & $52 \pm 8$ & $49 \pm 6$ & $44 \pm 5$ & $38 \pm 5$ \\
\hline Sham + IR + Iso & $100(99 \pm 11)$ & $73 \pm 7 *$ & $66 \pm 8^{*}$ & $59 \pm 7^{*}$ & $52 \pm 6 *$ \\
\hline TAC & $100(112 \pm 15)$ & $92 \pm 9$ & $89 \pm 8$ & $86 \pm 8$ & $81 \pm 7$ \\
\hline $\mathrm{TAC}+\mathrm{IR}$ & $100(110 \pm 14)$ & $50 \pm 8$ & $45 \pm 6$ & $41 \pm 6$ & $37 \pm 5$ \\
\hline $\begin{array}{l}\text { TAC + IR + Iso } \\
\text { IVEDP (mmHa) }\end{array}$ & $100(108 \pm 16)$ & $52 \pm 9$ & $46 \pm 7$ & $42 \pm 7$ & $39 \pm 7$ \\
\hline Sham & $5 \pm 1$ & $7 \pm 1$ & $8 \pm 1$ & $10 \pm 2$ & $11 \pm 2$ \\
\hline Sham $+\mathbb{R}$ & $5 \pm 1$ & $37 \pm 8$ & $33 \pm 7$ & $31 \pm 6$ & $31 \pm 6$ \\
\hline Sham + IR + Iso & $5 \pm 1$ & $24 \pm 6 *$ & $22 \pm 5^{*}$ & $21 \pm 5^{*}$ & $20 \pm 5^{*}$ \\
\hline TAC & $5 \pm 1$ & $7 \pm 1$ & $9 \pm 1$ & $11 \pm 2$ & $13 \pm 2$ \\
\hline $\mathrm{TAC}+\mathrm{IR}$ & $5 \pm 1$ & $42 \pm 9$ & $33 \pm 8$ & $30 \pm 7$ & $31 \pm 4$ \\
\hline TAC + IR + Iso & $5 \pm 1$ & $39 \pm 9$ & $31 \pm 6$ & $28 \pm 6$ & $30 \pm 5$ \\
\hline
\end{tabular}

controls, which is also consistent with a recent study ${ }^{15}$. However, several studies ${ }^{15,16}$ have shown that the infarct size was larger in hypertensive hypertrophied hearts exposed to IR in spontaneously hypertensive stroke-prone rats, although TAC induced ventricular hypertrophy, the extent of which was similar to ventricular hypertrophy in spontaneously hypertensive stroke-prone rats. The reason for the difference between spontaneously hypertensive stroke-prone rats and rats with TAC remains unclear, although different features between the two models of ventricular hypertrophy (duration of pressure overload) are possibly involved.

Apoptosis is a process of programmed cell death acomponied by morphological changes of cell. In this study we used TUNEL staining to measure myocardial apoptosis. Interestingly, we found that delayed isoflurane preconditioning significantly reduced the number of TUNEL-positive cell in sham-operated rat hearts, however, such anti-apoptotic effect was lost in hypertrophied hearts pretreated with isoflurane. In addition to morphological evaluation, we also aeesessed the Cleaved Caspase- 3 activity by immunoblotting. Caspase- 3 is the most important member of the caspase family and mediatess apoptotic signal translation pathway and the level of activated Caspase-3 indicates apoptosis of cells. We found that the Cleaved Caspase- 3 was significantly reduced in sham-operated hearts but not hypertrophied hearts exposed to delayed isoflurane peconditioning in the setting of IR, implying that the weakening of antiapoptotic effect in hypertrophied myocardium.

Even though eNOS and its produced NO were suggest to trigger and mediate delayed myocardial protection induced by isoflurane preconditioning in rabbits exposed to $\mathrm{IR}^{17}$, the iNOS produced NO also seems to be the key of delayed anesthetic preconditioning in rats $^{4,18}$. The observed variations in the source of NO may be related, at least in part, to the animal species (rats vs. rabbits) in which the studies were conducted and deserves further investigation. In our present study, we measured the expression of myocardial iNOS and eNOS to determine whether they are related to the delayed cardioprotection of isoflurane preconditioning in rat hearts. Here we demonstrated that the myocardial iNOS expression and the production

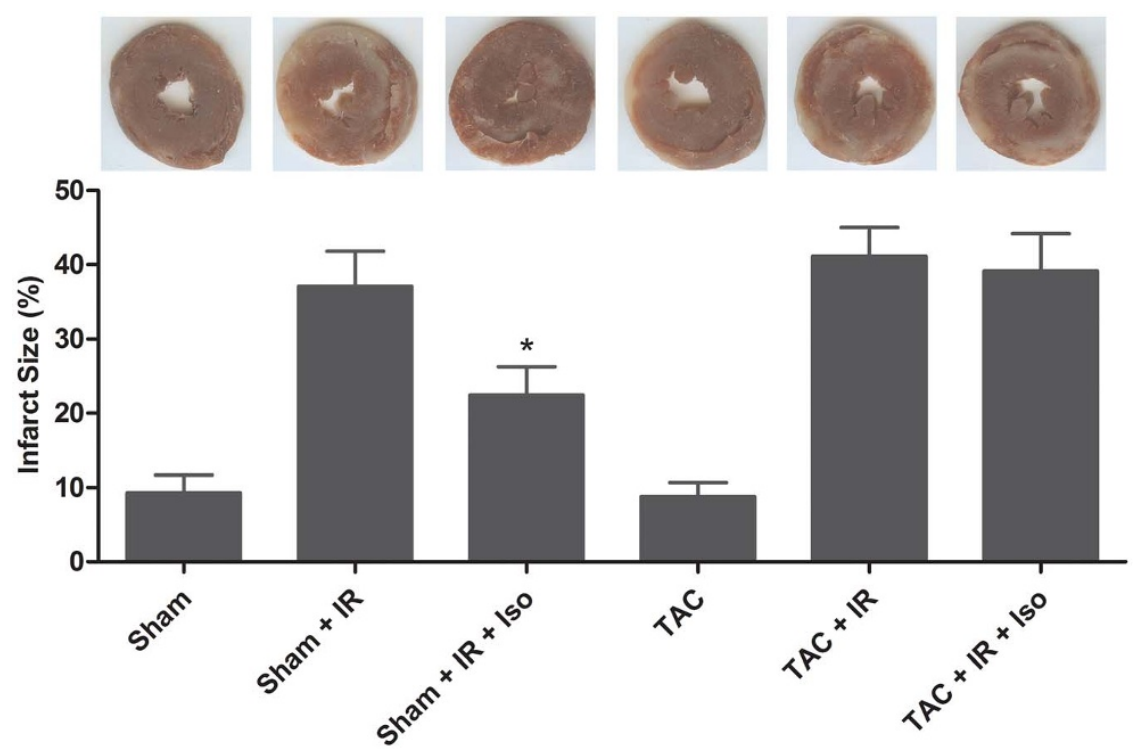

Figure 1 Effects of delayed isoflurane preconditioning on infarct size expressed as a percentage of total area of the myocardium in rat hearts exposed to ischemia-reperfusion. IR: ischemia reperfusion; Iso: Isoflurane; TAC: Transverse aortic constriction. Data are mean \pm SD, $\mathrm{n}=6 \mathrm{hearts} /$ group. $* \mathrm{P}<0.05$ vs. Sham + IR. 


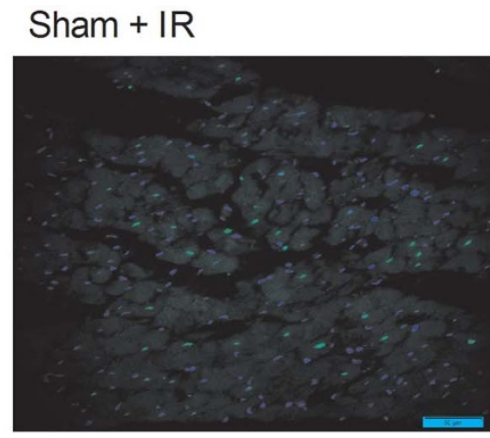

$T A C+I R$
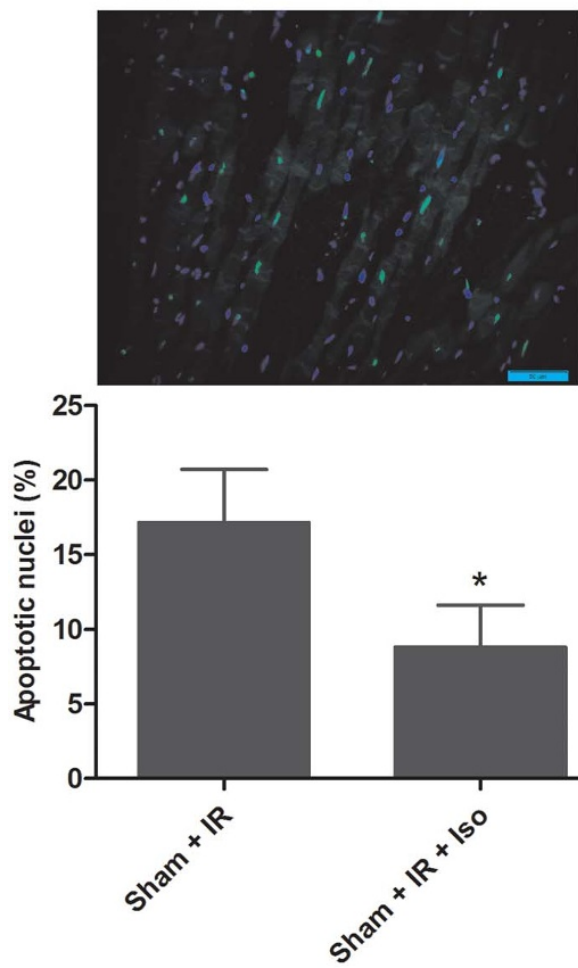

Sham + IR + Iso

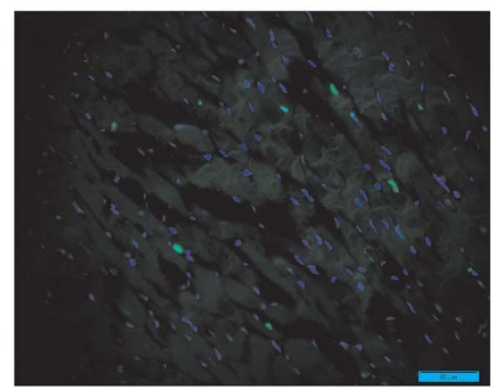

$T A C+I R+I S O$
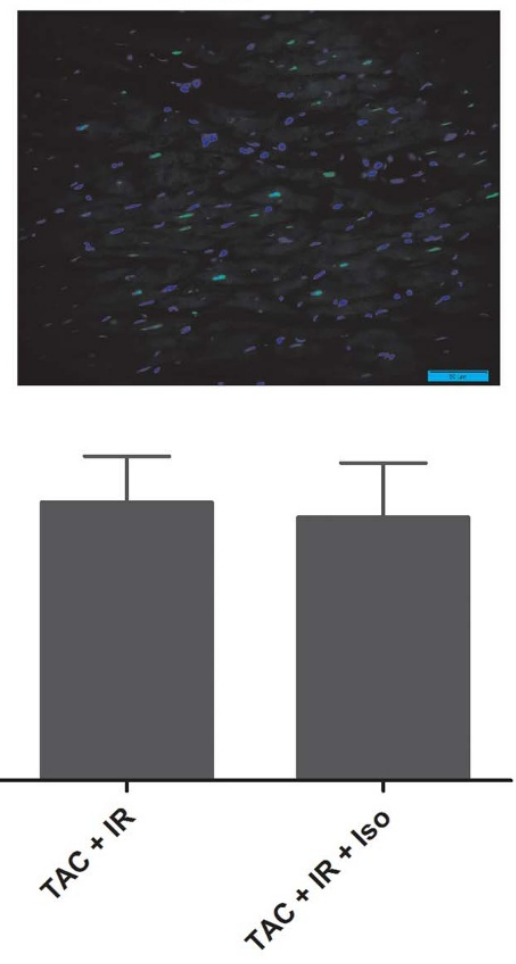

Figure 2 Effects of delayed isoflurane preconditioning on apoptosis expressed as a percent of total nuclei in tissue sections from rat hearts exposed to ischemia-reperfusion (TUNEL staining, $400 \times$ ). IR: ischemia reperfusion; Iso: Isoflurane; TAC: Transverse aortic constriction. Data are mean \pm SD, $\mathrm{n}=6$ hearts/group. ${ }^{*} \mathrm{P}<0.05$ vs. Sham + IR.

of NO was upregulated by delayed isoflurane preconditioning. And previous studies have demonstrated that the delayed cardioprotection of isoflurane preconditioning against IR injuries was abrogated by iNOS inhibitor $1400 \mathrm{~W}$ in rat hearts exposed to $\mathrm{IR}^{4,18}$. Nonetheless, the expression of eNOS was not affect by delayed isoflurane preconditioning. These results indicate that iNOS, but not eNOS, plays a pivotal role in the delayed cardioprotection of isoflurane preconditioning at least in the current model we adopted, which is also consistent with several previous studies ${ }^{4,18}$. Interestingly, such cardioprotection of delayed isoflurane preconditioning and upregulation of myocardial iNOS expression were both lost in hypertrophied hearts.

COX-2 is downstream of iNOS in delayed preconditioned myocardium and iNOS reduces myocardial injury by recruiting COX-2 in the setting of $\mathrm{IR}^{1,2}$. COX-2 and its major arachidonic acid products were shown to mediate late anesthetic preconditioning-induced myocardial protection ${ }^{4,5,19}$. The administration of celecoxib 2.5 hours before prolonged coronary artery occlusion and reperfusion, but not 30 minutes before isoflurane, abolished the cardioprotection associated with remote exposure to the volatile anesthetic ${ }^{5}$. Isoflurane exposure produces time-dependent increases of COX-2 protein expression and activity concomitant with a reduction in myocardial necrosis in rat hearts ${ }^{20}$. In our current study, we demonstrated that COX-2 activity was significantly upregulated by delayed isoflurane preconditioning in the healthy rat hearts subjected to IR. And previous studies have demonstrated that the delayed cardioprotection of isoflurane preconditioning against IR injuries was abrogated by COX-2 inhibitor in rat hearts exposed to $\mathrm{IR}^{20}$. Thus, these results indicate that COX-2 plays an obligatory role in delayed anesthetic cardioprotection in normal rats. Nonetheless, the upregulation of myocardial COX-2 expression was not found in hypertrophied hearts. If, as mentioned above, the upregulation of iNOS and COX-2 play a pivotal role in the infarct-sparing effect of delayed isoflurane preconditioning, we reasoned that the loss of cardioprotection in hypertrophied myocardium, at least in part, be attributed to the dysfunction of iNOS/COX-2 signal.

The findings provided here are translationally important in that they determined whether delayed anesthetic cardioprotection occurs in animals with hypertrophied myocardium. However, there are several limitations in our current work. First, although TUNEL staining is the most widely used method to detect apoptosis in the heart, its specificity has also been challenged due to its relatively high falsepositive rate ${ }^{21,22}$. Therefore, cautious interpretation of the staining is needed and apoptosis demonstrated by multiple criteria may be 
A

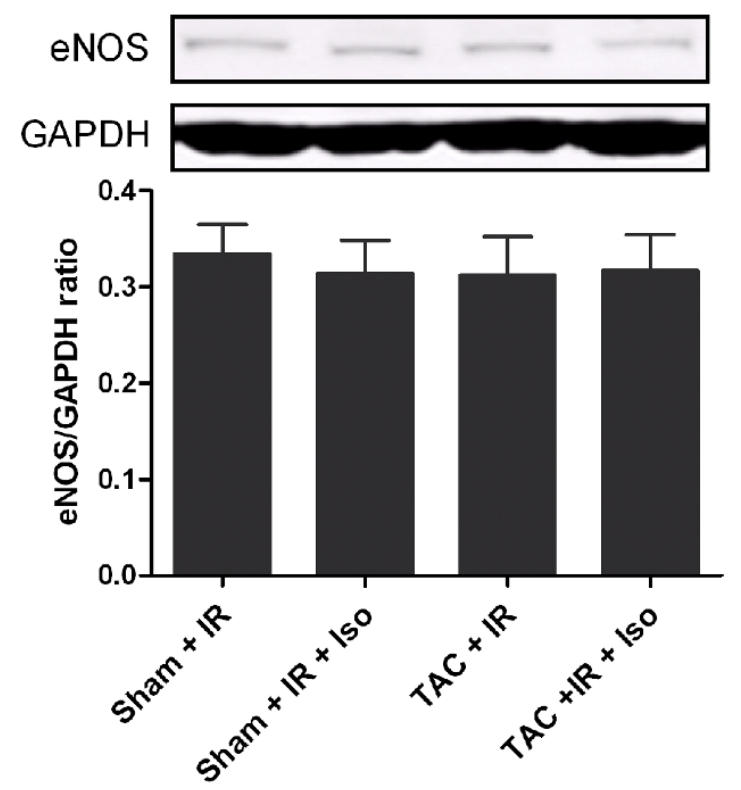

C

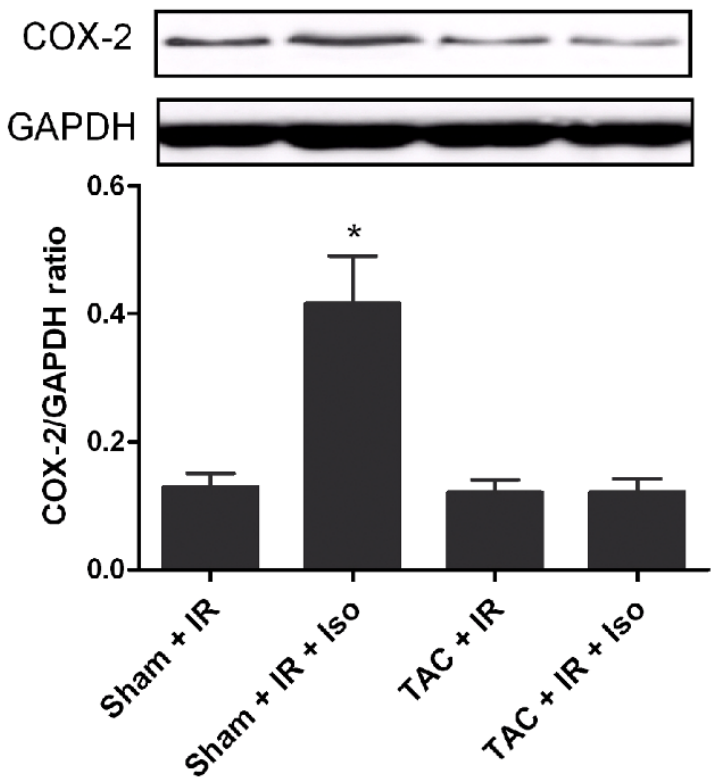

B

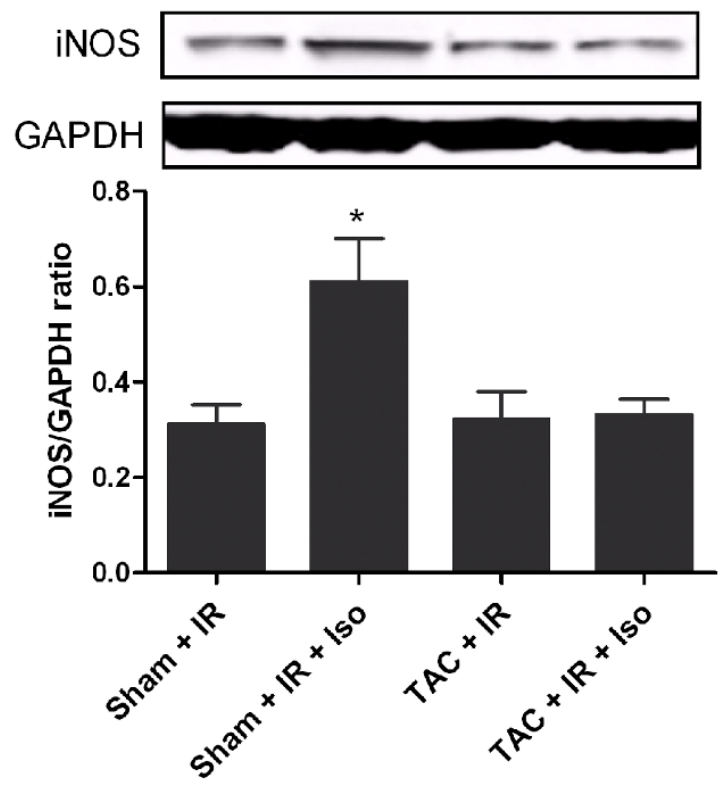

D
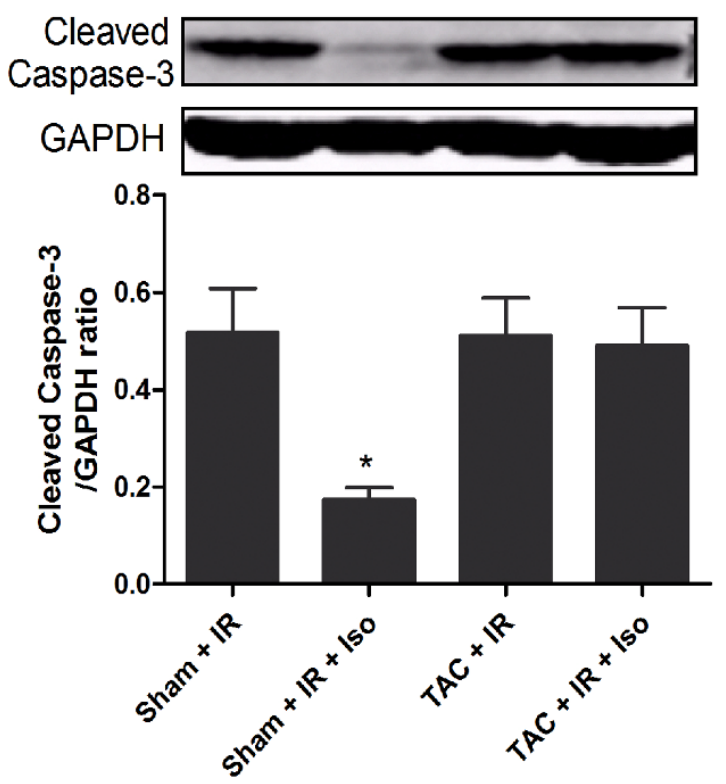

Figure $3 \mid$ Effects of delayed isoflurane preconditioning on the expression of eNOS (A), iNOS(B), COX-2 (C), Cleaved Caspase-3 (D) in rat hearts exposed to ischemia-reperfusion (IR). IR: ischemia reperfusion; Iso: isoflurane; TAC: Transverse aortic constriction. Data are mean \pm SD, $\mathrm{n}=6$ hearts/ group. ${ }^{*} \mathrm{P}<0.05$ vs. Sham + IR.

more appropriate in the future study. Second, the loss of delayed cardioprotection of isoflurane preconditioning in hypertrophied rat hearts might also be due to changes in oxidative stress ${ }^{23,24}$, which were not explored in the current study. Second, although ventricular hypertrophy may be associated with conditions other than hypertension, including myocardial infarction, anemia, aortic valve disease, hyperthyroidism, obesity, and renal disease, hypertension is the most common cause of ventricular hypertrophy ${ }^{25}$, which indicates that the hypertrophied rat heart model we used may not fully simulate the complex clinical setting of ventricular hypertrophy, so our results may apply only to the effect of ventricular hypertrophy on delayed anesthetic cardioprotection in a limited clinical setting.

In summary, our results showed that isoflurane preconditioning exerted delayed cardioprotection against IR injury in normal rats; this was blocked by ventricular hypertrophy potentially via interfering with iNOS expression and downstream COX-2 activation.

\section{Methods}

Animals. All of the animals were treated according to the guidelines of the Guide for the Care and Use of Laboratory Animals (United States National Institutes of Health). The Laboratory Animal Care Committee of Nanjing Medical University approved all experimental procedures and protocols. All efforts were made to minimize the number of animals used and their suffering. The rats were housed in polypropylene cages, and the room temperature was maintained at $22^{\circ} \mathrm{C}$, with a 12-hour light-dark cycle. Six-week-old male Sprague-Dawley rats, weighing 130-180 g, were used for all experiments.

In vivo experimental design. Rats were anesthetized with sodium pentobarbital ( $50 \mathrm{mg} / \mathrm{kg}$ intraperitoneally), then TAC was performed by the method of Perlini et $\mathrm{a}^{26}$ with slight modifications. The suprarenal portion of the aorta was exposed and a blunted 22-gauge needle placed adjacent to the aorta. A ligature (5-0 silk) was snugly tied around both the aorta and the needle. The needle was then removed, leaving the internal diameter of the aorta approximately equal to that of the needle. Shamoperated animals had an untied ligature placed in the same location. After the operation, the animals were housed under controlled environmental conditions and fed with standard pellet chow for 8 weeks. The diastolic left ventricular posterior wall 


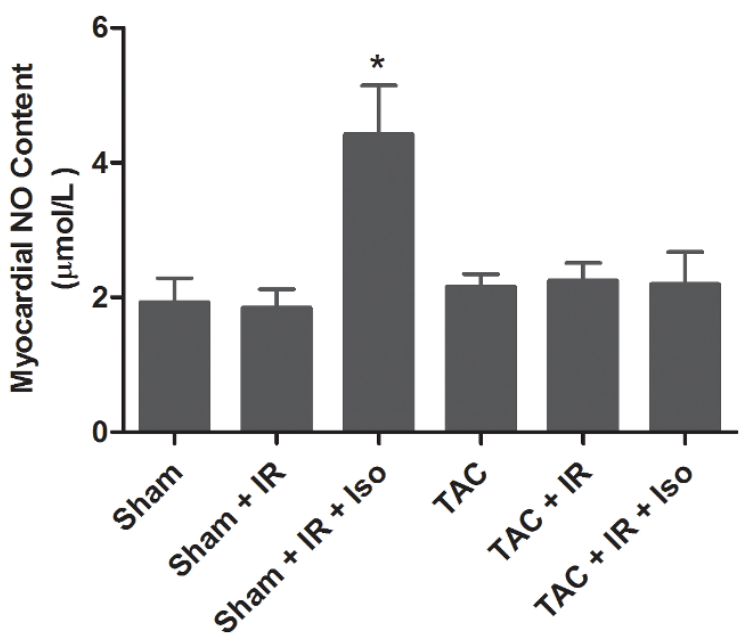

Figure $4 \mid$ Effects of delayed isoflurane preconditioning on the production of NO in rat hearts exposed to ischemia-reperfusion (IR). IR: ischemia reperfusion; Iso: isoflurane; TAC: Transverse aortic constriction. Data are mean $\pm \mathrm{SD}, \mathrm{n}=6$ hearts/group. ${ }^{*} \mathrm{P}<0.05$ vs. Sham + IR.

thickness was assessed by using the Vevo 2100 system (VisualSonics, Toronto, Canada) with a 21-MHz transducer. Then sham-operated and TAC-rats were placed in an induction chamber for treatment with $2.1 \%(\mathrm{v} / \mathrm{v})$ isoflurane (Maruishi Pharmaceutical Co, Ltd, Osaka, Japan) in $33 \% \mathrm{O}_{2}$ by spontaneous ventilation for $1 \mathrm{~h}$ and then housed in room air overnight before isolated heart perfusion ${ }^{4,9,20}$. An anesthetic gas monitor Vamos (Dräger Medical AG \& Co. KG, Lübeck, Germany) was used to continuously monitor the concentration of isoflurane.

Isolated heart perfusion protocol. All hearts were allowed to equilibrate for $30 \mathrm{~min}$ and subjected to $40 \mathrm{~min}$ of global ischemia by stopping the K-H buffer perfusion $\left(37^{\circ} \mathrm{C}\right)$ followed by $120 \mathrm{~min}$ of reperfusion ${ }^{27,28}$. The experiments were conducted as follows: 1) sham group (Sham): sham-operated rats pretreated with $33 \% \mathrm{O}_{2}$ were perfused for $190 \mathrm{~min}$; 2) sham-operated ischemia reperfusion group (Sham + IR): sham-operated rats pretreated with $33 \% \mathrm{O}_{2}$ were subjected to 40 min of global ischemia and 120 min of reperfusion; 3) sham-operated isoflurane preconditioning group (Sham + IR + Iso): sham-operated rats pretreated with $2.1 \%(\mathrm{v} / \mathrm{v})$ isoflurane were subjected to $40 \mathrm{~min}$ of global ischemia and $120 \mathrm{~min}$ of reperfusion; 4)

Transverse aortic constriction group (TAC): TAC-rats pretreated with $33 \% \mathrm{O}_{2}$ were perfused for $190 \mathrm{~min}$; 5) Transverse aortic constriction plus ischemia reperfusion group (TAC + IR): TAC-rats pretreated with $33 \% \mathrm{O}_{2}$ were subjected to $40 \mathrm{~min}$ of global ischemia and $120 \mathrm{~min}$ of reperfusion only; 6) Transverse aortic constriction plus isoflurane preconditioning group (TAC + IR + Iso): TAC-rats pretreated with $2.1 \%(\mathrm{v} / \mathrm{v})$ isoflurane were subjected to $40 \mathrm{~min}$ of global ischemia and $120 \mathrm{~min}$ of reperfusion.

Isolated heart preparation. After the rats were anesthetized by pentobarbital sodium $(50 \mathrm{mg} / \mathrm{kg}$ intraperitoneally) and then heparinized by heparin $(300 \mathrm{U} / \mathrm{kg}$ intraperitoneally) for $5 \mathrm{~min}$, hearts were isolated rapidly, placed in ice-cold KrebsHenseleit (K-H) buffer and mounted on the Langendorff apparatus. Retrograde perfusion was initiated under constant pressure $(70 \mathrm{~mm} \mathrm{Hg})$ with gassed $\left(95 \% \mathrm{O}_{2}\right.$, $5 \% \mathrm{CO}_{2}$ ) K-H buffer containing (in mM) $118 \mathrm{NaCl}, 4.7 \mathrm{KCl}, 1.2 \mathrm{KH}_{2} \mathrm{PO}_{4}, 1.2 \mathrm{MgSO}_{4}$, $1.25 \mathrm{CaCl}_{2}, 25.0 \mathrm{NaHCO}_{3}$, and 11.0 glucose, $\mathrm{pH} 7.4$, at $37^{\circ} \mathrm{C}$. Hearts met the exclusion criteria (time to perfusion $>3 \mathrm{~min}$; coronary flow $<10 \mathrm{ml} / \mathrm{min}$ or $>28 \mathrm{ml} / \mathrm{min}$; arrhythma duration $>3 \mathrm{~min}$; heart rate $<70$ beats per min or $>400$ beats per min; Left ventricular developed pressure $<70 \mathrm{mmHg}$ or $>130 \mathrm{mmHg}$ ) for Langendorff perfused heart were not included in the study ${ }^{29}$.

Hemodynamic measurements. For the measurements of LV pressure, a latex fluidfilled balloon was inserted into the left ventricle through the left atrial appendage and the balloon catheter was linked to a pressure transducer connected to a data acquisition system (RM6240; Chengdu Biological Instruments, Chengdu, China). The LV systolic pressure (LVSP), LV end diastolic pressure (LVEDP, adjusted to between 4 and $8 \mathrm{~mm} \mathrm{Hg}$ before ischemia), LV developed pressure (LVDP $=$ LVSP LVEDP) and heart rate (HR) were continuously recorded.

Determination of infarct size. Myocardial infarct size was measured by 2,3,5triphenyltetrazolium chloride (TTC, Sigma-Aldrich, St. Louis, MO) staining at the end of $120 \mathrm{~min}$ of reperfusion ${ }^{27}$. Briefly, the hearts were frozen at $-20^{\circ} \mathrm{C}$ for $2-3 \mathrm{~h}$, cut into 2-mm-thick slices, incubated with $1 \%$ TTC solution for $5 \mathrm{~min}$ at $37^{\circ} \mathrm{C}$. All slices were then scanned together, and the areas of myocardial infarction (pale) in each slice were analyzed by Image J 1.37 (National Institutes of Health, Bethesda, $\mathrm{MD})$. The infarct size was expressed as percentage of the total slice area.
Detection of Myocardial Apoptosis. Apoptosis was assessed using the TUNEL method. At the end of reperfusion, the hearts were fixed in $4 \%$ paraformaldehyde and embedded in paraffin for TUNEL staining. The heart tissue sections were stained using an in situ cell death detection kit (POD; Roche Diagnostics Corp, Indianapolis, IN, USA), following the manufacturer's protocol using a fluorescence microscope. Ten microscopic fields $(400 \times)$ from each section were assayed by counting TUNELpositive cells. The percentage of TUNEL-positive nuclei (green nuclei) was calculated.

Immunoblotting. At the end of reperfusion, the samples were taken from ischemic zone. The expression of myocardial Cleaved Caspase-3 (Cell Signaling Technology, Beverly, MA, USA) was determined by immunoblotting ${ }^{27}$. A separate cohort of rats was used for the study of eNOS, iNOS and COX-2 (Cell Signaling Technology, Beverly, MA, USA) expression. Briefly, sham-operated and TAC-rats were treated with $33 \% \mathrm{O}_{2}$ or $2.1 \%$ isoflurane for $1 \mathrm{~h}$, then the left ventricular tissue samples were harvested for immunoblotting $24 \mathrm{~h}$ later ${ }^{27}$.

Measurement of myocardial NO content. NO content was evaluated by measuring nitrite according to the Griess methods ${ }^{30}$. In brief, hearts were harvested $24 \mathrm{~h}$ after isoflurane exposure, and the LV tissue samples was frozen in liquid nitrogen, homogenized in buffer and centrifuged at 14,000 g for $20 \mathrm{~min}$. The level of NO was measured using the commercially available total nitric oxide assay Kit (Beyotime Institute of Biotechnology, Haimen, China) according to the manufacturer's instructions.

Statistical analysis. Data are shown as mean $\pm \mathrm{SD}$. The data for mean blood pressure, the ratio of heart weight: body weight and left ventricular posterior wall thickness were analyzed using the unpaired Student's $t$ test. For hemodynamic data, repeatedmeasures analysis of variance with post hoc Student-Newman-Keuls test for multiple comparisons was used to evaluate differences over time between groups. All other data were analyzed by one-way ANOVA following Student-Newman-Keuls post hoc test. A value of $\mathrm{P}<0.05$ was considered to be statistically significant. All statistical analyses were performed using SPSS 13.0 (SPSS Inc., Chicago, IL).

1. Hausenloy, D. J. \& Yellon, D. M. The second window of preconditioning (SWOP) where are we now? Cardiovasc Drugs Ther 24, 235-254 (2010).

2. Pagel, P. S. \& Hudetz, I. A. Delayed Cardioprotection by Inhaled Anesthetics. J Cardiothoracic Vasc Anesth 25, 1125-1140 (2011).

3. Shinmura, K. et al. Inducible nitric oxide synthase modulates cyclooxygenase-2 activity in the heart of conscious rabbits during the late phase of ischemic preconditioning. Circ Res 90, 602-608 (2002).

4. Wakeno-Takahashi, M., Otani, H., Nakao, S., Imamura, H. \& Shingu, K. Isoflurane induces second window of preconditioning through upregulation of inducible nitric oxide synthase in rat heart. Am J Physiol Heart Circ Physiol 289, H2585-H2591 (2005).

5. Tanaka, K. et al. Isoflurane produces delayed preconditioning against myocardial ischemia and reperfusion injury: role of cyclooxygenase-2. Anesthesiology 100, 525-531 (2004).

6. Guo, Y. et al. The COX-2/PGI2 receptor axis plays an obligatory role in mediating the cardioprotection conferred by the late phase of ischemic preconditioning. PLoS One 7, e41178 (2012).

7. Ehring, T. et al. Attenuation of myocardial stunning by the ACE inhibitor ramiprilat through a signal cascade of bradykinin and prostaglandins but not nitric oxide. Circulation 90, 1368-1385 (1994).

8. Jalowy, A., Schulz, R., Dorge, H., Behrends, M. \& Heusch, G. Infarct size reduction by AT1-receptor blockade through a signal cascade of AT2-receptor activation, bradykinin and prostaglandins in pigs. J Am Coll Cardiol 32, 1787-1796 (1998).

9. Xie, H. et al. The changes of technetium-99m-labeled annexin- $\mathrm{V}$ in delayed anesthetic preconditioning during myocardial ischemia/reperfusion. Mol Biol Rep 41, 131-137 (2013).

10. Tang, X. L., Stein, A. B., Shirk, G. \& Bolli, R. Hypercholesterolemia blunts NO donor-induced late preconditioning against myocardial infarction in conscious rabbits. Basic Res Cardiol 99, 395-403 (2004).

11. Tang, X. L. et al. Hypercholesterolemia abrogates late preconditioning via a tetrahydrobiopterin-dependent mechanism in conscious rabbits. Circulation 112, 2149-2156 (2005).

12. Vogt, M., Motz, W., Scheler, S. \& Strauer, B. E. Disorders of coronary microcirculation and arrhythmias in systemic arterial hypertension. Am J Cardiol 65, 45G-50G (1990).

13. Rakusan, K. \& Wicker, P. Morphometry of the small arteries and arterioles in the rat heart: effects of chronic hypertension and exercise. Cardiovasc Res 24, 278-284 (1990).

14. Prisant, L. M. Hypertensive heart disease. J Clin Hypertens (Greenwich) 7, 231-238 (2005).

15. Yano, T. et al. Hypertensive hypertrophied myocardium is vulnerable to infarction and refractory to erythropoietin-induced protection. Hypertension $\mathbf{5 7}$, 110-115 (2011)

16. Dai, W., Simkhovich, B. Z. \& Kloner, R. A. Ischemic preconditioning maintains cardioprotection in aging normotensive and spontaneously hypertensive rats. Exp Gerontol 44, 344-349 (2009). 
17. Chiari, P. C. et al. Role of endothelial nitric oxide synthase as a trigger and mediator of isoflurane-induced delayed preconditioning in rabbit myocardium. Anesthesiology 103, 74-83 (2005).

18. Chen, C. H., Chuang, J. H., Liu, K. \& Chan, J. Y. Nitric oxide triggers delayed anesthetic preconditioning-induced cardiac protection via activation of nuclear factor kappaB and upregulation of inducible nitric oxide synthase. Shock 30, 241-249 (2008).

19. Tonkovic-Capin, M. et al. Delayed cardioprotection by isoflurane: role of K(ATP) channels. Am J Physiol Heart Circ Physiol 283, H61-H68 (2002).

20. Feng, J. et al. Cardiac remodelling hinders activation of cyclooxygenase-2, diminishing protection by delayed pharmacological preconditioning: role of HIF1 alpha and CREB. Cardiovasc Res 78, 98-107 (2008).

21. Labat-Moleur, F. et al. TUNEL apoptotic cell detection in tissue sections: critical evaluation and improvement. J Histochem Cytochem 46, 327-334 (1998).

22. Ding, B. et al. Left ventricular hypertrophy in ascending aortic stenosis mice: anoikis and the progression to early failure. Circulation 101, 2854-2862 (2000).

23. Kupai, K. et al. Cholesterol diet-induced hyperlipidemia impairs the cardioprotective effect of postconditioning: role of peroxynitrite. Am J Physiol Heart Circ Physiol 297, H1729-1735 (2009).

24. Iliodromitis, E. K. et al. Simvastatin in contrast to postconditioning reduces infarct size in hyperlipidemic rabbits: possible role of oxidative/nitrosative stress attenuation. Basic Res Cardiol 105, 193-203 (2010).

25. Diamond, J. A. \& Phillips, R. A. Hypertensive heart disease. Hypertens Res 28 , 191-202 (2005).

26. Perlini, S. et al. Sympathectomy or doxazosin, but not propranolol, blunt myocardial interstitial fibrosis in pressure-overload hypertrophy. Hypertension 46, 1213-1218 (2005).

27. Zheng, Z. et al. Gender-related difference of sevoflurane postconditioning in isolated rat hearts: focus on phosphatidylinositol-3-kinase/Akt signaling. J Surg Res 170, e3-e9 (2011).

28. Ma, L. L. et al. Hypertrophied myocardium is refractory to sevoflurane-induced protection with alteration of reperfusion injury salvage kinase/glycogen synthase kinase $3 \beta$ signals. Shock 40, 217-221 (2013).
29. Bell, R. M., Mocanu, M. M. \& Yellon, D. M. Retrograde heart perfusion: the Langendorff technique of isolated heart perfusion. J Mol Cell Cardiol 50, 940-950 (2011).

30. Wang, M. et al. Glucose regulated proteins 78 protects insulinoma cells (NIT-1) from death induced by streptozotocin, cytokines or cytotoxic T lymphocytes. Int J Biochem Cell Biol 39, 2076-2077 (2007).

\section{Acknowledgments}

This work was supported by the National Natural Science Foundation of China (No. 81301608), National Natural Science Foundation of China (No. 81401633), Zhejiang Provincial Natural Science Foundation of China (No. LY14H150005), and Zhejiang Provincial Medical Technology Foundation of China (No. 2014KYA171)

\section{Author contributions}

L.M., J.L. and F.K. analyzed data; L.M., F.K., F.G., L.X. and R.S. performed research; L.M., H.G., F.K. and B.H. wrote the paper.

\section{Additional information}

Competing financial interests: The authors declare no competing financial interests.

How to cite this article: Ma, L. et al. Ventricular hypertrophy blocked delayed anesthetic cardioprotection in rats by alteration of iNOS/COX-2 signaling. Sci. Rep. 4, 7071; DOI:10.1038/srep07071 (2014).

This work is licensed under a Creative Commons Attribution-NonCommercialNoDerivs 4.0 International License. The images or other third party material in this article are included in the article's Creative Commons license, unless indicated otherwise in the credit line; if the material is not included under the Creative Commons license, users will need to obtain permission from the license holder in order to reproduce the material. To view a copy of this license, visit http:// creativecommons.org/licenses/by-nc-nd/4.0/ 